\title{
How Does Closure of Tympanic Membrane Perforations Affect Hearing and Middle Ear Mechanics? - An Evaluation in a Patient Cohort and Temporal Bone Models
}

\author{
Röösli, C ; Sim, J H ; Chatzimichalis, M ; Huber, A M
}

\begin{abstract}
OBJECTIVE: This study aimed to determine how tympanic membrane (TM) perforations and their closure, using a paper-patch technique, affect middle-ear mechanics and, thus, conductive hearing for different sizes of the TM perforation. STUDY DESIGN: Temporal bone (TB) study and prospective clinical trial. SETTING: Tertiary referral center. PATIENTS: Nine patients with chronic otitis media for more than 3 months. INTERVENTION: The TM perforations were closed with a paper patch in all 9 patients. In 5 of 9 patients, myringoplasty was performed. Matching TM perforations were created in a TB model $(n=8)$ and closed using the paper patch. MAIN OUTCOME MEASURES: Air-bone gap was measured in all 9 patients of the patient cohort with TM perforations before and after closure and in 5 patients after myringoplasty. Stapes velocity and sound pressure difference between the ear canal and middle-ear cavity were measured in TBs with intact TM, with TM perforations, and with the perforations closed by the paper patch. All measurements in the patient cohort and TBs were performed for different sizes of TM perforations to determine if the effects varied as a function of size. RESULTS: Degree of the air-bone gap differed as a function of size of the TM perforations and its recovery after closure, and myringoplasty was independent of the size of the TM perforation in the frequency range of 0.25 to $4 \mathrm{kHz}$. In the TB measurements, although pressure difference across the TM was almost fully recovered by closing the perforation with a paper patch, recovery of the stapes motion was limited at frequencies above $4.5 \mathrm{kHz}$ for larger sizes of TM perforations. CONCLUSION: Hearing loss caused by TM perforations depends on the size of the perforation. Hearing returns almost completely across the frequency range after closure except above $4 \mathrm{kHz}$ for larger perforations. This is because the structural damage caused by large TM perforations cannot be completely restored by application of a paper patch.
\end{abstract}

DOI: https://doi.org/10.1097/MAO.0b013e31824296ee

Posted at the Zurich Open Repository and Archive, University of Zurich

ZORA URL: https://doi.org/10.5167/uzh-57388

Journal Article

Originally published at:

Röösli, C; Sim, J H; Chatzimichalis, M; Huber, A M (2012). How Does Closure of Tympanic Membrane Perforations Affect Hearing and Middle Ear Mechanics? - An Evaluation in a Patient Cohort and Temporal Bone Models. Otology and Neurotology, 33(3):371-378.

DOI: https://doi.org/10.1097/MAO.0b013e31824296ee 


\section{How does closure of tympanic membrane perforations affect hearing and middle ear mechanics?}

- An evaluation in a patient cohort and temporal bone models

Christof Röösli, MD; Jae Hoon Sim, PhD; Michailis Chatzimichalis, MD, PhD; Alex M Huber, MD

Clinic of Otorhinolaryngology - Head and Neck Surgery, University Hospital Zurich, Zurich, Switzerland

Reprint requests and correspondence:

Christof Röösli, MD

Clinic of Otorhinolaryngology, Head and Neck Surgery, University Hospital Zurich

Frauenklinikstrasse 24

$\mathrm{CH}-8091$ Zurich

Switzerland

Telephone: $\quad++41442555850$

E-Mail: christof.roeoesli@usz.ch

Short running title: TM perforation and middle ear mechanics

Keywords: Otitis media chronica simplex, tympanic membrane perforation, temporal bone, stapes velocity, pressure differences 


\section{Abstract}

Objective: This study aimed to determine how tympanic membrane (TM) perforations and their closure using a paper-patch technique affect middle-ear mechanics and thus conductive hearing, for different sizes of the TM perforation.

Study design: Temporal bone (TB) study and prospective clinical trial

Setting: Tertiary referral center

Patients: Nine patients with chronic otitis media for more than 3 months.

Intervention: The TM perforations were closed with a paper patch in all nine patients. In 5 of 9 patients, myringoplasty was performed. Matching TM perforations were created in a temporal bone model $(n=8)$ and closed using the paper patch

Main outcome Measures: Air-bone gap (ABG) was measured in all 9 patients of the patient cohort with TM perforations before and after closure and in 5 patients after myringoplasty. Stapes velocity and sound pressure difference between the ear canal and middle-ear cavity were measured in TBs with intact TM, with TM perforations, and with the perforations closed by the paper patch. All measurements in the patient cohort and TBs were performed for different sizes of TM perforations to determine if the effects varied as a function of size.

Results: Degree of the ABG differed as a function of size of the TM perforations and its recovery following closure and myringoplasty was independent of the size of the TM perforation in the frequency range of 0.25 to $4 \mathrm{kHz}$. In the TB measurements, while pressure difference across the TM was almost fully recovered by closing the perforation with a paper patch, recovery of the stapes motion was limited at frequencies above $4.5 \mathrm{kHz}$ for larger sizes of TM perforations.

Conclusion: Hearing loss caused by TM perforations depends on the size of the perforation. Hearing returns almost completely across the frequency range following closure except above 4 $\mathrm{kHz}$ for larger perforations. This is because the structural damage caused by large TM perforations cannot be completely restored by application of a paper patch. 


\section{Introduction}

Chronic otitis media simplex is often encountered in clinical practice and has an incidence of 1 to $8.6 \%(1,2)$. The symptoms of tympanic membrane (TM) perforations, which usually include conductive hearing loss $(\mathrm{CHL})$ and recurrent infections that make repetitive local treatment necessary, vary widely from patient to patient. While $\mathrm{CHL}$ due to TM perforation can be minimal and not bothersome in some patients, it can reach 50 to $60 \mathrm{~dB} \mathrm{HL}$ in other patients thus impacting daily life. Furthermore, when the TM perforation is combined with additional middle-ear infections, it is difficult to determine how much of the total $\mathrm{CHL}$ is due to the TM perforation alone.

Previous theoretical (3), experimental $(4,5)$ and clinical $(6,7)$ data have shown that $C H L$ due to TM perforation increases with increasing perforation size, and is greater at lower than at higher frequencies. It was explained that a larger size perforation results in smaller sound pressure differences across the TM and thus in a larger $\mathrm{CHL}$.

Effects of the location of the TM perforation on conductive hearing have been an issue for the last several decades with contradictory results. Schmitt (8) described the differences of magnitude and phase of sound pressure between the round and oval window in patients with TM perforations as a reason for hearing loss. Perforations on a location directly lateral to the round window were considered to increase sound pressure around the round window to a larger extent than perforations on other locations of the TM, resulting in a reduction of sound pressure difference between the oval and round windows. Ahmad et al. (9) observed a larger CHL below $1500 \mathrm{~Hz}$ in patients with TM perforations on the posterior-inferior parts of the TM, and Masuda et al. (10) found the largest $\mathrm{CHL}$ in patients with TM perforations on the anterior-inferior quadrant compared to the other quadrants of the TM. By contrast, significant correlation between location of the TM perforation and $\mathrm{CHL}$ was not observed in other clinical $(6,7)$, and experimental studies $(5,11)$. Mehta et al. (6) also argued that wavelengths in the frequency range below $4 \mathrm{kHz}$ are larger than middle-ear dimensions; therefore, sound reaches the round and oval window simultaneously, independent of the location of the TM perforation.

Whether location of the TM perforation affects $\mathrm{CHL}$ or not, successful myringoplasty is presumed to re-establish sound pressure differences across the TM to the same state as before it 
occurred. If change in sound pressure differences across the TM is responsible for most of the $\mathrm{CHL}$, then it would be expected that a myringoplasty would restore hearing to normal postoperatively. However, this is not always the clinical reality, and $\mathrm{CHL}$ still remains significant in some patients despite closure of the TM perforation.

This study aimed to determine how TM perforation and its closure by a paper-patch technique affect middle-ear mechanics and thus conductive hearing, for different sizes of TM perforations. One hypothesis was that the closure of the TM perforation by a paper patch reverses the hearing loss caused by the TM perforation regardless of the size of the TM perforation. Hearing thresholds were measured in a prospective patient cohort with TM perforations, after paper patching the perforation, and post-operatively after myringoplasty. In temporal bone (TB) models, pressure difference between the middle-ear cavity and ear canal and stapes motion as representatives of conductive hearing were measured with either intact (or normal), perforated, or paper-patched TM status and compared to the results from the patient cohort. Though effects of location of the TM perforation were not directly investigated, they were discussed from the results in TB models.

\section{Material and Methods}

\section{Patient cohort}

Nine patients with otitis media chronica simplex (five right and four left ears) from May 2008 to August 2010 were included in the patient cohort of this study. The nine patients consisted of 7 men and 2 women with an average age of 49.3 years (ranging from 32 to 75 years). The study was approved by the ethical committee (Kantonale Ethikkomission Zürich, Nr. 727) and was performed according to the Declaration of Helsinki (12). Informed consent was obtained from all patients. Inclusion criterion was otitis media chronica simplex for more than 3 months, and exclusion criteria were acute infections, traumatic perforations, age below $18 \mathrm{yrs}$, or pregnancy.

Subjective hearing thresholds were measured for three different statuses of the TM: with the perforation open (all nine patients), immediately after closing the TM using a paper patch (all 
nine patients), and 12 months after closure by myringoplasty/spontaneous closure (five of nine patients). The paper patch was applied as an office procedure with the patient seated in an upright position. Local anaesthesia using a small piece of cotton soaked with $10 \%$ Lidocaine hydrochloridum anhydricum (Xylocain, AstraZeneca, London, UK) was placed in the external ear canal on the remaining TM. Next, a paper patch of about $66 \mu \mathrm{m}$ thickness (King size blue, Miquel Y Costa \& Miquel, Barcelona, Spain), consisting of cellulose, was placed temporarily on the TM such that it covered the perforation completely, and was removed after audiometry. A surgical intervention (myringoplasty) to close the TM perforation was performed on five of nine patients, with general anesthesia using facia of the temporalis muscle.

For audiometry, air-conduction (AC) testing was performed with standard supra-aural earphones. Both $A C$ and bone conduction $(B C)$ thresholds were measured at $0.25,0.5,1,2,3$, and $4 \mathrm{kHz}$, and the air-bone gap (ABG) was calculated from the measurements. Masking with narrow band noise at $60 \mathrm{~dB} \mathrm{HL}$ was applied to the non-test ear for all $\mathrm{BC}$ threshold measurements. Masking was used for $A C$ testing when the $A C$ threshold of the test ear differed from the $B C$ threshold of the non-test ear by more than $40 \mathrm{~dB}$.

The patients were classified into three categories according to the area ratio of the TM perforation to the total TM area: "small", less than $8 \%(n=5)$; "medium", between 8 and 25\% $(n=3)$; and "large" greater than $25 \%(n=1)$. A limit of $8 \%$ for small, and $25 \%$ for medium TM perforation was chosen, because it confined its location within the anterior-inferior quadrant, and the anterior half, respectively. The perforated TMs were photographed with a zero-degree optic. Using the projected photo, the ratio of the area of the TM perforation relative to the total TM area was determined using ImageJ software (NIH, Bethesda, MD, USA). The absolute size of the TM was assumed from the literature (13) to have a vertical diameter of $9.5 \mathrm{~mm}$ along the axis of the manubrium and a horizontal diameter of $8.5 \mathrm{~mm}$ along the axis passing through the tip of the manubrium and perpendicular to the vertical axis. The size of the TM perforation was calculated relatively based on the assumed size of the TM. Among the five patients who underwent myringoplasty, three patients belonged to the "small", one to the "medium", and one to the "large" categories. 


\section{Temporal Bone Measurements}

Stapes velocity and sound pressure in the ear canal as well as in the tympanic cavity were measured in eight fresh TBs from human cadavers (within 7 days after death) with intact, perforated, and paper-patched TMs in sequence, for the three different sizes of TM perforations that represented the three categories defined in the patient cohort. The perforations were made with a sickle knife and micro-scissors under magnification using a surgical microscope. The "small" and "medium" sized perforations were located in the inferior-anterior quadrant, and the "large" size perforation covered most of the inferior side (Fig. 1). The area of the TM perforations were approximately 2,10 , and $35 \%$ of the total TM area, for the "small", "medium", and "large" sizes, respectively. The paper patch was moistened with saline as needed and carefully applied to cover the perforation completely.

Three TBs were used only for the "small" size of the TM perforation and one only for the "medium" size. Two sizes of TM perforation were created in the other four TBs (one for the "small" and "large" sizes of the TM perforation, and three for the "medium" and "large" sizes). When the two sizes of the TM perforation were applied to a TB, measurements with the smaller size (including measurements with the perforation closed by the paper patch) were first conducted followed by the same procedures after creating the larger perforation. Consequently, four measurement sets for each perforation size category were obtained.

The donors of the eight TBs had an average age of 59 years (ranging from 23 to 76 years) and all measurements were done within one week from death. The human TBs were harvested within 24 hours after death, and were preserved in $0.1 \%$ Cialit (sodium-2-ethylmercurimer- captobenzoxazol-5-carbonide) solution at $4^{\circ} \mathrm{C}$ until the measurements were performed. The preparation of the temporal bones has been described previously (14). Briefly, the external ear canal was drilled down to $2 \mathrm{~mm}$ from the tympanic annulus. An artificial external ear canal consisting of a plastic tube with a length of $1.5 \mathrm{~cm}$ and a volume of $0.5 \mathrm{ml}$ was attached to the rim and sealed acoustically using silicon and cast. Additionally, a pressure tube was inserted in the mastoid cavity and the opening of the mastoidectomy cavity was sealed by application of cast, Vaseline, and a 
transparent anti-reflecting glass plate. All TBs were periodically moistened with a physiological saline solution to prevent drying during preparation.

The loudspeaker (ER-2, Etymotic Research, USA) and the microphone probe (ER-14C, Etymotic Research, USA) of the probe microphone assembly (ER-7C, Etymotic Research, USA) were placed in the artificial external ear canal near the TM to provide acoustic stimuli and monitor the ear-canal pressure. Harmonic signals of 32 different frequencies in the range of 0.25 to $8 \mathrm{kHz}$ were generated by the PSV board (V.8.6, Polytec GmbH, Germany), and were delivered to the loudspeaker individually via an amplifier (RMX 850, QSC Audio Products LLC, USA), resulting in a sound pressure level in the artificial ear canal of $88 \mathrm{~dB}$ SPL and up to $105 \mathrm{~dB}$ SPL for higher frequencies to obtain a good signal-to-noise ratio. The frequency steps were $100 \mathrm{~Hz}$ up to $1.8 \mathrm{kHz}$, $200 \mathrm{~Hz}$ up to $3 \mathrm{kHz}, 300$ or $400 \mathrm{~Hz}$ up to $4 \mathrm{kHz}$, and $500 \mathrm{~Hz}$ from 4 to $8 \mathrm{kHz}$.

As our PSV board had only two measurement channels, motion of the stapes and pressure in the artificial ear canal were first measured, then the pressures in the middle-ear cavity and in the artificial ear canal were measured. Repeated measurements of pressure in the ear canal were checked and were consistent. The motions of the stapes were measured at five points near the center of the footplate using a scanning laser Doppler interferometry (SLDI) system (OFV-3001, Polytec $\mathrm{GmbH}$ ), and the measurements at the five points were averaged. Because these five points showed almost the same magnitudes and phases, the averaged motion from the five points was considered as a translational motion component of the stapes footplate. A signal-to-noise ratio of $20 \mathrm{~dB}$ or higher was achieved for all measurement points included in this analysis.

The measurement of the stapes motion was corrected by an angle between the laser beam of the SLDI system and the stapes footplate, which was obtained using micro-CT techniques. The details were described in our previous study (14).

\section{Statistics}

The Student's t-test was performed to see significant differences in ABG between the paperpatched $(n=9)$ and post-operative group $(n=5)$ in the patient cohort without the perforation size distinguished (unpaired t-test), and between the intact and paper-patched TMs in the temporal 
bone models (one-tailed, paired t-test). The $p$ values less than 0.05 were considered as the level of significance.

\section{Results}

\section{Patient cohort}

Table 1 lists sizes, locations and categories of the TM perforations, as described in the Methods section, for the nine patients. The vertical diameter ranged between 0.51 and $5.8 \mathrm{~mm}$, the horizontal diameter between 0.32 and $4.5 \mathrm{~mm}$, and the area ratio of the TM perforation to the total TM between 0.17 and $34.5 \%$. The perforation was located in the anterior-inferior quadrant in 5 patients (56\%), overlapping in the anterior-inferior and posterior-inferior quadrants in 3 patients (33\%), and including all four quadrants in one patient (11\%). As a consequence, all perforations were partially in the anterior half and none was found isolated in the posterior half. Five of the nine patients underwent myringoplasty, Of these 5 patients, 3 belonged to the "small", 1 to the "medium", and 1 to the "large" categories of TM perforations.

Figure 2 illustrates air-bone gaps (ABGs) with the TM perforations and after closure by paper patch, for the three categories. The largest ABGs were at $0.25 \mathrm{kHz}$, and the smallest at 2 $\mathrm{kHz}$ for all categories. The amount of $\mathrm{ABG}$ at $0.25 \mathrm{kHz}$ varied with size category ranging from approximately $20 \mathrm{~dB}$ for the "small" up to $50 \mathrm{~dB}$ for the "medium" and "large" sizes. Generally, the larger the size of the TM perforation, the greater the ABG. After application of the paper patch, the $A B G$ s were closed, and the recovered $A B G$ s were similar for the three categories. In summary, although the degree of $A B G$ differed with the sizes of the TM perforations, the recovered ABG after the paper-patch was similar independently of the size of the TM perforation, in the considered frequency range of 0.25 to $4 \mathrm{kHz}$.

The combined ABGs for all patients are plotted in Fig. 3 for both conditions. The ABGs after closing the perforation by paper patch was approximately $15 \mathrm{~dB}$ at $0.25 \mathrm{kHz}$, and $0 \mathrm{~dB}$ at $2 \mathrm{kHz}$. The average ABG across frequency was $8.2 \mathrm{~dB}$. There were no significant differences $(p>0.05)$ 
between the paper-patched $(n=9)$ and post-operative (with myringoplasty, $n=5)$ groups in the considered frequency range of 0.25 to $4 \mathrm{kHz}$.

\section{Temporal Bone Measurements}

Figure 4 displays mean values in pressure differences between the ear canal and middleear cavity (A), and velocity of the stapes $(B)$, where each of the quantities was calculated relative to the ear canal pressure for measurements with intact TMs $(n=8)$. The mean pressure in the middle-ear cavity was less than $10 \%$ of the mean pressure in the ear canal across frequency except in the $1-\mathrm{kHz}$ region, where the pressure measurements had a large deviation. Variation of pressure in the middle-ear cavity (relative to the pressure in the ear canal) was $2.7 \pi$ radians (i.e., slightly less than 1.5 cycles). The normalized mean stapes velocity was maximum around $0.7 \mathrm{kHz}$ and minimum around $4 \mathrm{kHz}$ with a phase variation of $2.3 \pi$ radians.

Measurements with the perforated TMs are shown in Fig. 5. The pressure difference was reduced by a large amount, especially below $3 \mathrm{kHz}$. Even negative values in the pressure difference were observed for some cases. The chamber of the artificial ear canal with TM perforation is also presumed to contribute to the negative values as a kind of Helmholtz resonator. The reduction was largest near $0.5 \mathrm{kHz}$ for the small size of the perforation and near $1 \mathrm{kHz}$ for the medium and large sizes. Variability between individual measurements was largest near the frequency of greatest reduction in both pressure and phase and was similar to the corresponding variation in the measurements with the intact TMs. The normalized stapes velocity showed almost the same magnitude compared to the corresponding magnitude in the intact TMs for small and medium sizes of TM perforation, but significantly reduced magnitude for the large sized perforation.

After the perforations were closed by the paper patch, the pressure difference and stapes velocity recovered to values similar to those in the intact-TM condition (Fig. 6). Only magnitude of the stapes velocity with the large size TM perforation failed to recover fully in the high frequencies. This is apparent in Fig. 7, which represents changes in magnitudes of the pressure difference and stapes velocity after paper patch compared to the corresponding magnitudes with the intact TMs. Significant difference $(p<0.05)$ between the two TM conditions (i.e., intact and paper-patched) 
was observed only for the stapes velocity with the large size TM perforation at frequencies above $4.5 \mathrm{kHz}$ (marked by * in Fig. 7B).

\section{Discussion}

Sound pressure difference across the TM is known to be the primary factor accounting for motion of the TM and thereby the ossicular chain in the middle ear (4). We expected that the larger the size of a TM perforation, the larger the effects on hearing because of a greater reduction in this sound pressure difference. This was confirmed by our results both from the patient cohort and the TB measurement. In the TB measurement, both the sound pressure difference across the TM and motion of the stapes were reduced. The reduction in pressure decrease across the TM was large below $1 \mathrm{kHz}$ for the "small" size and below $2 \mathrm{kHz}$ for the "medium" and "large" categories. That is to say, as perforation size increased, there were larger changes in sound pressure difference over a wider frequency range. These results are in agreement with clinical, theoretical, and experimental findings reported in the literature $(3-6,15-19)$. Based on our results, slight hearing losses for patients with "small" TM perforations are limited to frequencies below 0.5 $\mathrm{kHz}$. Therefore, when a pancochlear $A B G$ occurs involving the high frequencies or the $A B G$ is larger than expected based on the perforation size, then possible additional middle-ear pathologies such as a dehiscence of the ossicular chain or a malleus or stapes fixation should be considered. Another factor that can cause an unexpectedly large ABG is a small middle ear space. It has been shown that the volume of the middle ear space can have an effect on sound transmission of up to $35 \mathrm{~dB} \mathrm{HL}$ (3-5), and the same size of TM perforation can cause different degrees of hearing loss as a result. As the focus in our study was on the effect of closing the perforation with a paper patch, the changes in sound pressure difference across the TM and stapes motion between the normal and paper-patched TM were compared individually for each TB. Therefore, effects of middle-ear volume were not measured separately. Our surgical approach produced a mastoid cavity that was sealed with a glass cover and had approximately the same volume for all temporal bones. 
Comparing the effect of the paper patch in the patient cohort with the TB measurements shows a larger residual $A B G$ in the patient cohort. For determination of $A B G$ with the patient cohort, the non-test ear was masked by narrow band noise at $60 \mathrm{~dB} \mathrm{HL}$ to prevent it from influencing results of $\mathrm{BC}$ hearing thresholds of the tested ear. However, it is known that level of masking affects $A C$ and $B C$ thresholds, especially $B C$ thresholds (20). While over-masking of the non-test ear results in increased $B C$ thresholds of the tested ear due to unintentional masking of the tested ear, under-masking of the non-tested ear results in decreased $\mathrm{BC}$ thresholds of the tested ear due to bilateral summation. Consequently, the increased and decreased BC thresholds result in smaller and larger ABGs, respectively than true ABGs. Masking level could have influenced thresholds in the patient cohort and be the reason for less recovery of the ABG (i.e. more residual $A B G$ ) than expected recovery from the TB measurement.

The method of using a paper patch to close a TM perforation to predict hearing outcome is known as the paper-patch test (21), and was reported to be limited to perforations of greater than $50 \%$ of the TM membrane (22). Though application of the paper patch was bothersome for some patients with larger TM perforations, the largest TM perforation in the patient cohort was only 34\% of the total TM area, and a good seal could be achieved. The effects of covering the TM perforation with a paper patch were similar to those of the myringoplasty for our patient cohort, even with different material properties between the paper and the fascia used for TM reconstruction as reported by others (21).

While the ABG measurement in the patient cohort could be calculated only up to $4 \mathrm{kHz}$ due to technical limitations of the BC vibrator at higher frequencies, the TB measurements were extended to $8 \mathrm{kHz}$. Above $4.5 \mathrm{kHz}$, the pressure difference across the TM recovered almost fully by paper-patch application, independent of the size of the TM perforation, but recovery of stapes motion was limited for the "large" TM perforation, which involved most of the inferior area of the TM. It was observed that stapes motion was significantly reduced above $4.5 \mathrm{kHz}$ with the large perforation, though pressure difference across the TM was not significant in that frequency range. These results cannot be explained if reduction in pressure difference across the TM is the cause of hearing loss in patients with TM perforations. O'Connor et al. (23) reported that radial collagen in 
the TM plays an important role in sound transmission through the middle ear above $4 \mathrm{kHz}$. They argued that sound transmission with the TM perforation covered would result in a different type of sound transmission than occurs in normal ears because the radial collagen in damaged TMs cannot be restored by simply closing the TM perforation. We conclude from our results that the large perforation caused structural damage of the TM that was not fully restored by closing it, resulting in limited recovery of high-frequency hearing.

\section{Conclusion}

The ABG in patients with TM perforation depends on the size of the perforation, therefore, $a$ pancochlear or large ABG in patients with a small TM perforation and an aerated middle-ear can be suggestive of additional pathologies in the middle ear.

While pressure difference across the TM was almost fully restored by closing the perforation with a paper patch regardless of the size of the perforation, recovery in motion of the stapes was limited above $4.5 \mathrm{kHz}$ for the large TM perforation. Such a large perforation, which included most of the inferior area of the TM, is presumed to cause structural damage that cannot be restored by the paper patch. 


\section{References}

1. Gladstone HB, Jackler RK, Varav K. Tympanic membrane wound healing. An overview. Otolaryngol Clin North Am 1995; 28: 913-32.

2. Griffin WL. A retrospective study of traumatic tympanic membrane perforations in a clinical practice. Laryngoscope 1979; 89: 261-82.

3. Voss SE, Rosowski JJ, Merchant SNet al. Middle-ear function with tympanic-membrane perforations. II. A simple model. J Acoust Soc Am 2001; 110: 1445-52.

4. Voss SE, Rosowski JJ, Merchant SNet al. Middle-ear function with tympanic-membrane perforations. I. Measurements and mechanisms. J Acoust Soc Am 2001; 110: 1432-44.

5. Voss SE, Rosowski JJ, Merchant SNet al. How do tympanic-membrane perforations affect human middle-ear sound transmission? Acta Otolaryngol 2001; 121: 169-73.

6. Mehta RP, Rosowski JJ, Voss SEet al. Determinants of hearing loss in perforations of the tympanic membrane. Otol Neurotol 2006; 27:136-43.

7. Anthony WP, Harrison CW. Tympanic membrane perforation. Effect on audiogram. Arch Otolaryngol 1972; 95: 506-10.

8. Schmitt, H. Uber die bedeutung der schalldrucktransformation und der schallprotektion für die hörschwelle (About the importance of sound pressure transformation and the acoustic protection for the hearing threshold). Acta Oto-Laryngol 1958; 49: 71-80.

9. Ahmad SW, Ramani GV. Hearing loss in perforations of the tympanic membrane. $J$ Laryngol Otol 1979; 93: 1091-8.

10. Matsuda Y, Kurita T, Ueda Yet al. Effect of tympanic membrane perforation on middle-ear sound transmission. J Laryngol Otol Supp/ 2009: 81-9.

11. Voss SE, Rosowski JJ, Merchant SNet al. Non-ossicular signal transmission in human middle ears: Experimental assessment of the "acoustic route" with perforated tympanic membranes. J Acoust Soc Am 2007; 122: 2135-53.

12. WMA Declaration of Helsinki - Ethical Principles for Medical Research Involving Human Subjects. Available at: http://www.wma.net/en/30publications/10policies/b3/index.html, Acessed August 25, 2010. 
13. Wever EG, Lawrence M. Physiological Acoustics. Princetown, N.J.: Princetown University Press. 1954

14. Sim JH, Chatzimichalis M, Lauxmann M. et al. Complex stapes motions in human ears. J Assoc Res Otolaryngol 2010; 11: 329-41.

15. Bigelow DC, Swanson PB, Saunders, JC. The effect of tympanic membrane perforation size on umbo velocity in the rat. Laryngoscope 1996; 106: 71-6.

16. McArdle FE, Tonndorf J. Perforations of the tympanic membrane and their effects upon middle-ear transmission. Arch. Ohren. Nasen Kehlkopfheilkd 1968; 192: 145-62.

17. Kruger B, Tonndorf J. Middle ear transmission in cats with experimentally induced tympanic membrane perforations. J Acoust Soc Am 1977; 61: 126-32.

18. Kruger B, Tonndorf J. Tympanic membrane perforations in cats: Configurations of losses with and without ear canal extensions. J Acoust Soc Am 1978; 63: 436-41.

19. Gan RZ, Cheng T, Dai C, Yang F, Wood MW. Finite element modeling of sound transmission with perforations of tympanic membrane. J Acoust Soc Am 2009; 126(1): 243-53.

20. Ito T, Röösli C, Kim CJ, Sim JH, Huber AM, Probst R. Bone conduction thresholds and skull vibration measured on the teeth during simulation at different sites on the human head. Audiology and Neurotology 2011; 16: 12-22.

21. Schuknecht HF. Surgery of the Ear and Temporal Bone. New York: Raven Press, 1993. 18.

22. Wada H, Kobayashi T, Tachizaki H. Diagnosis of middle ear disease with eardrum perforation by a newly developed sweep frequency measuring apparatus. Audiology 1992; 31(3):132-9.

23. O'Connor KN, Tam M, Blevins NH, Puria S, Tympanic membrane collagen fibers: a key to high-frequency sound conduction. Laryngoscope 2008; 118: 483-90. 


\section{$\underline{\text { Tables and Figure Captions }}$}

Table 1. Sizes of TM perforations in nine patients of the patient cohort.

Figure 1. Three sizes of the TM perforation made in TB models. "Small" (left), "medium" (middle), and "large" (right) sizes.

Figure 2. Air-bone gaps (ABGs) in the patient cohort with TM perforations $(A)$ and with the perforations closed by paper patch (B). Vertical lines indicate one standard deviation.

Figure 3. Combined mean air-bone gaps (ABGs) in the patient cohort with TM perforation, with the perforation closed by paper patch, and post-operatively after myringoplasty. Vertical lines indicate one standard deviation.

Figure 4. Magnitude (left) and phase (right) in pressure difference between the ear canal and middle ear cavity $(A)$ and stapes motion $(B)$ for TB measurements with intact TMs $(n=8)$. The magnitudes and phases were calculated relative to the magnitude and phase of the ear-canal pressure. Vertical lines indicate one standard deviation.

Figure 5. Magnitude (left) and phase (right) in pressure difference between the ear canal and middle ear cavity (A) and stapes motion (B) for TB measurements with perforated TMs ( $n=3$ for "small", n=4 for "medium" and "large" sizes). Vertical lines indicate one standard deviation.

Figure 6. Magnitude (left) and phase (right) in pressure difference between the ear canal and middle ear cavity $(A)$ and stapes motion (B) for TB measurements with TM perforations closed by paper patch. Vertical lines indicate one standard deviation.

Figure 7. Change in magnitudes of pressure difference between the ear canal and middle ear cavity $(A)$ and stapes motion $(B)$ after paper patch, compared to the corresponding magnitudes in TB measurements with intact TMs. In the frequency range (from 5 to $8 \mathrm{kHz}$ ) marked by *, stapes motions with paper-patched TM were significantly different $(p<0.05)$ from stapes motion with the intact TM. 
Table 1

\begin{tabular}{c|cc|c|c} 
& \multicolumn{2}{|c|}{ Diameter [mm] } & $\begin{array}{c}\text { Perforation area / } \\
\text { TM area [\%] }\end{array}$ & $\begin{array}{c}\text { Category of TM } \\
\text { perforation size }\end{array}$ \\
\cline { 2 - 3 } & Vertical & Horizontal & 4.77 & Small \\
Patient 1 & 2.27 & 1.78 & 9.03 & Medium \\
Patient 2 & 3.17 & 2.91 & 2.08 & Small \\
Patient 3 & 1.20 & 1.23 & 8.52 & Medium \\
Patient 4 & 2.92 & 2.57 & 34.50 & Large \\
Patient 5 & 5.81 & 4.50 & 13.85 & Medium \\
Patient 6 & 4.08 & 3.03 & 2.72 & Small \\
Patient 7 & 1.58 & 1.30 & 5.68 & Small \\
Patient 8 & 2.03 & 2.08 & 0.17 & Small \\
Patient 9 & 0.51 & 0.32 & 9.04 & \\
\hline Average & 2.62 & 2.19 & &
\end{tabular}


Figure 1

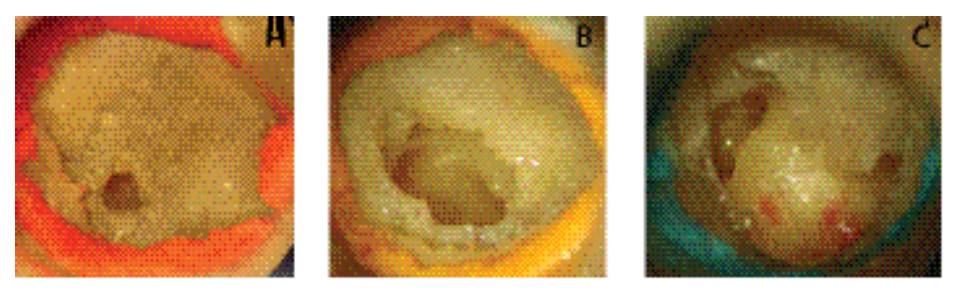


Figure 2

A

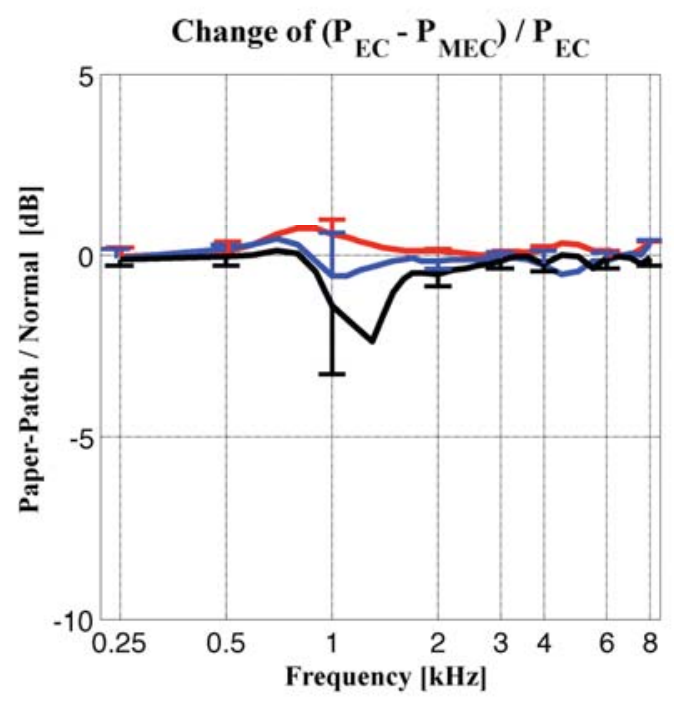

B

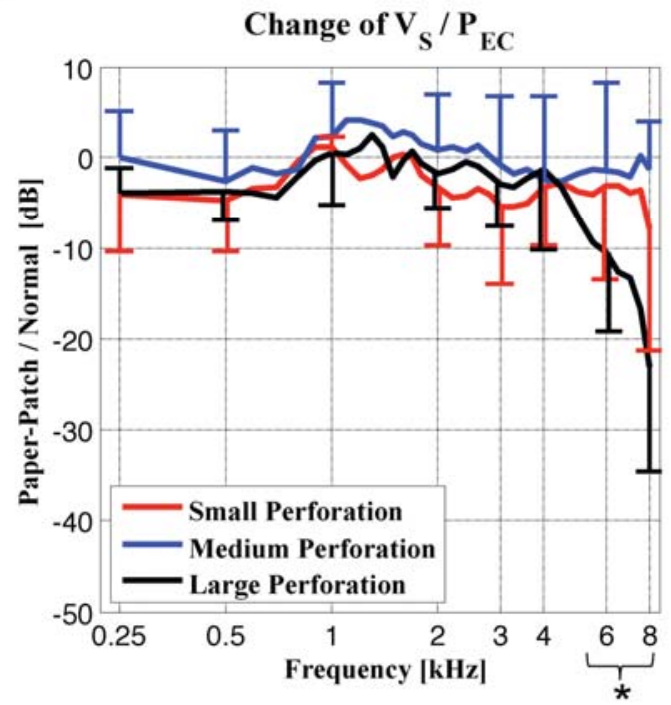


Figure 3

A

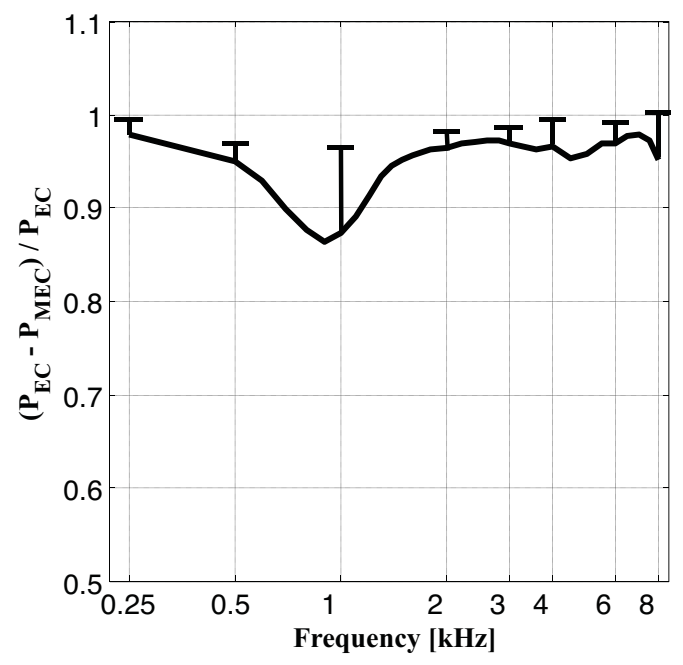

B

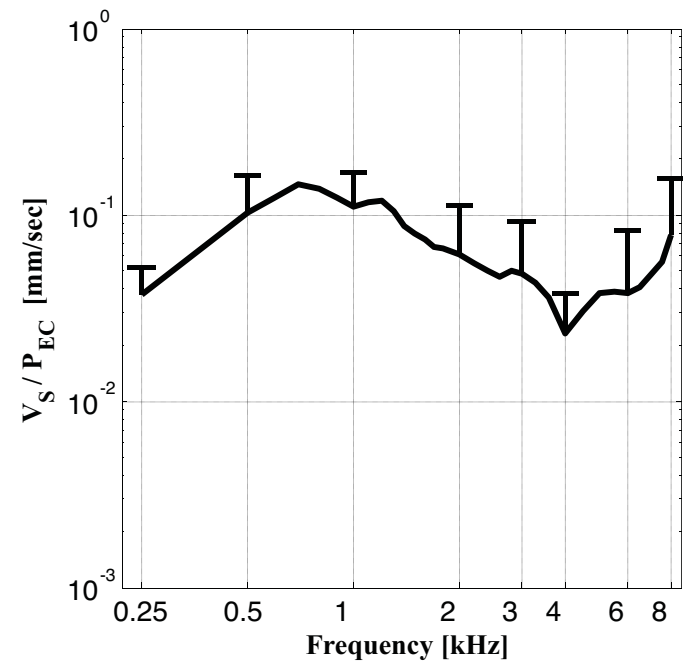

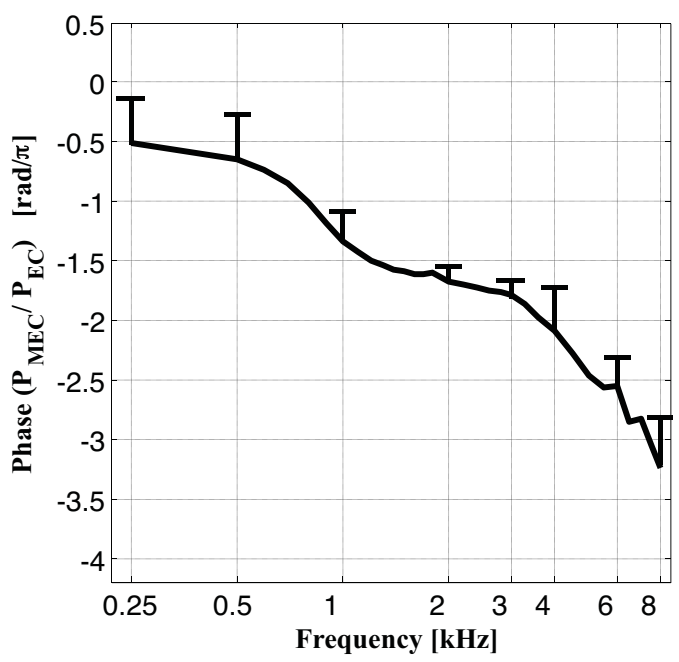

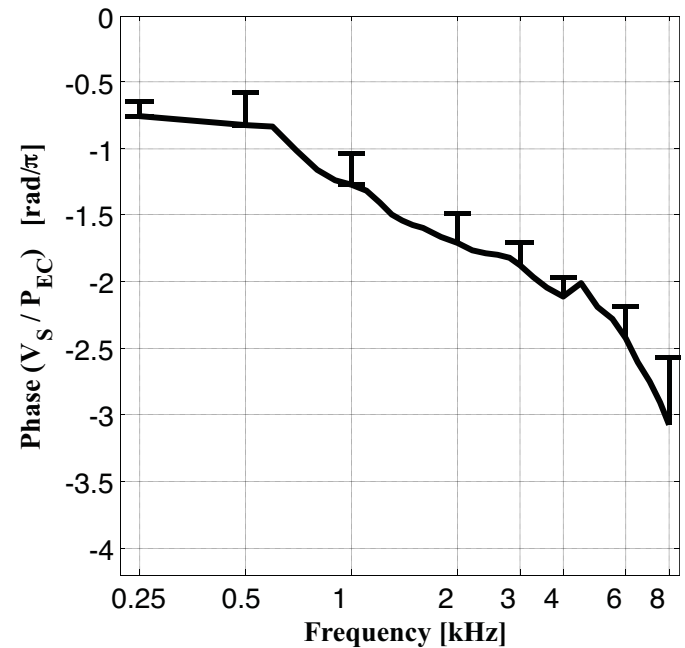


Figure 4

A

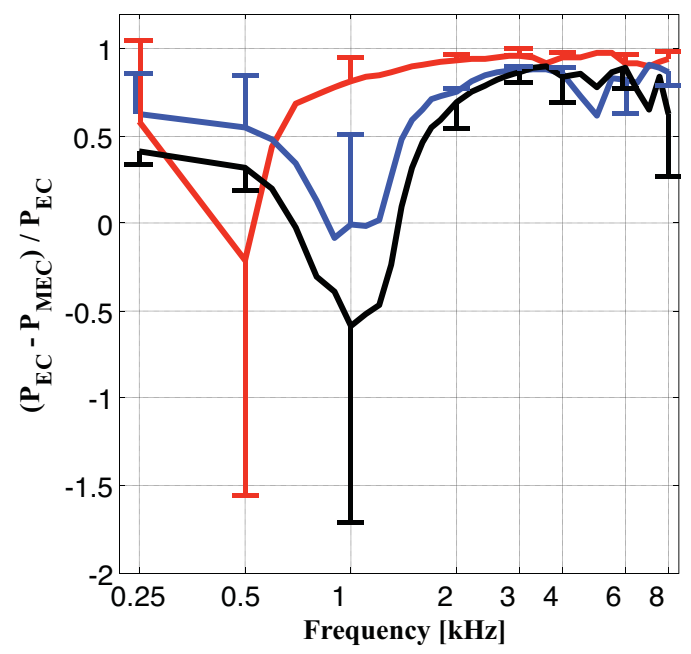

B

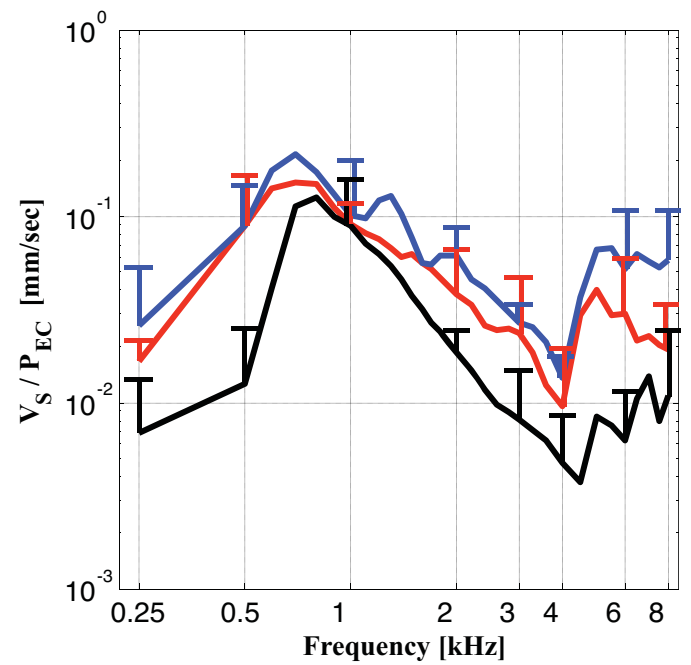

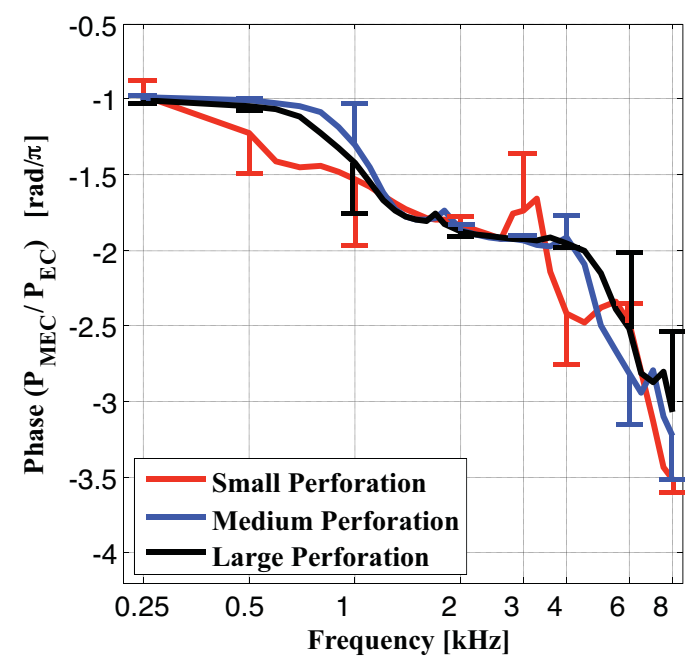

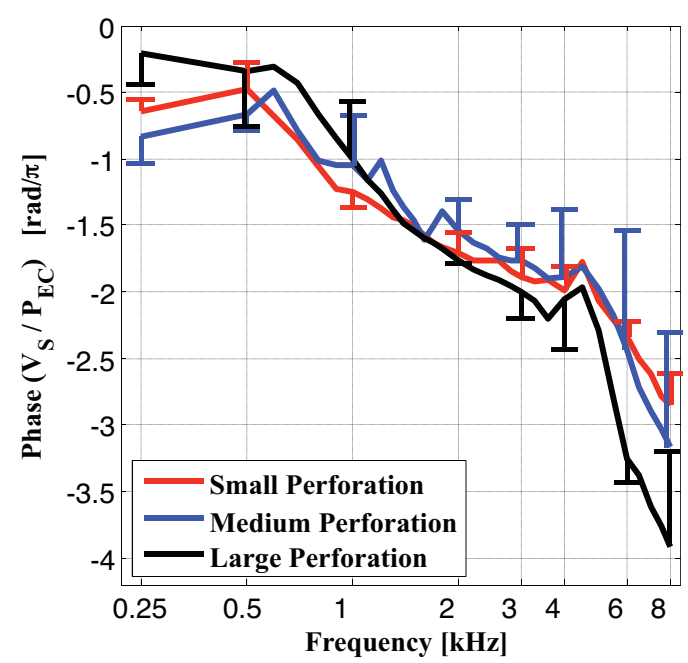


Figure 5

A

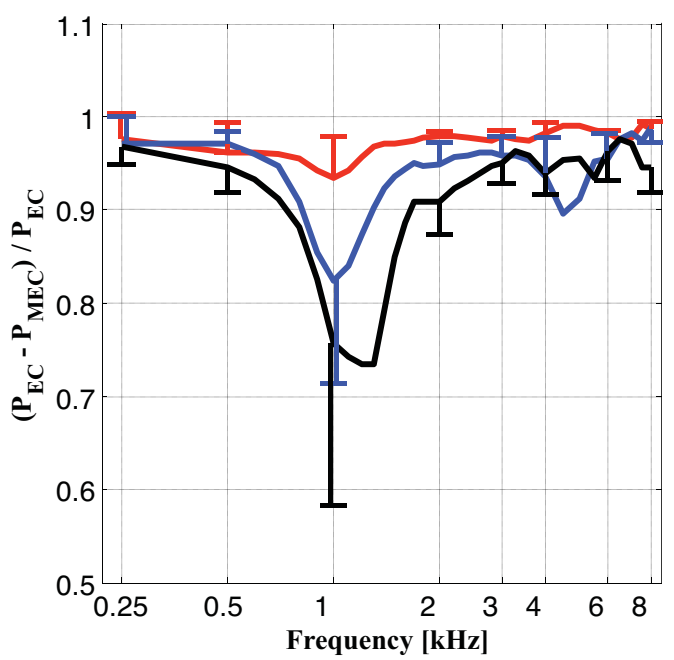

B

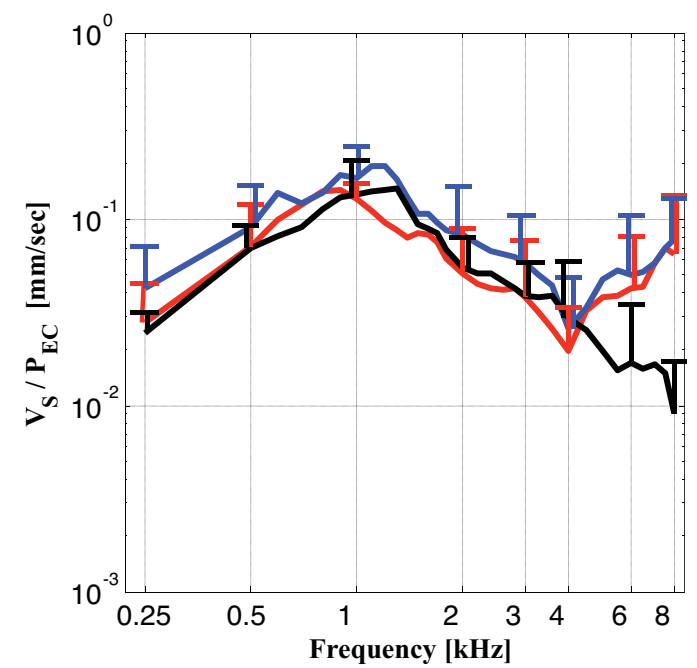

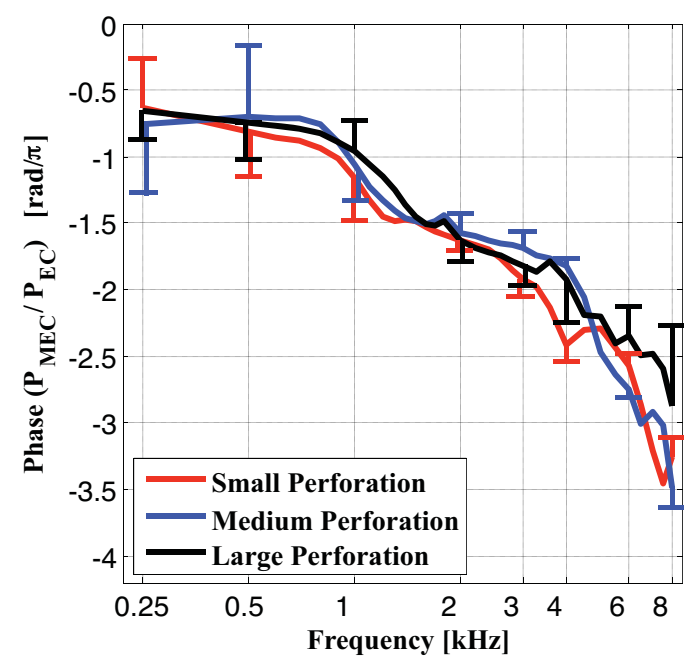

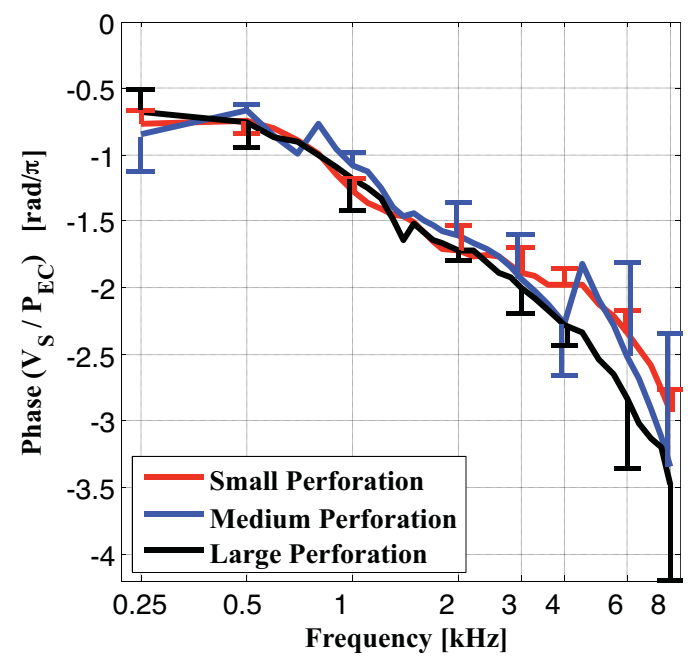


Figure 6

A

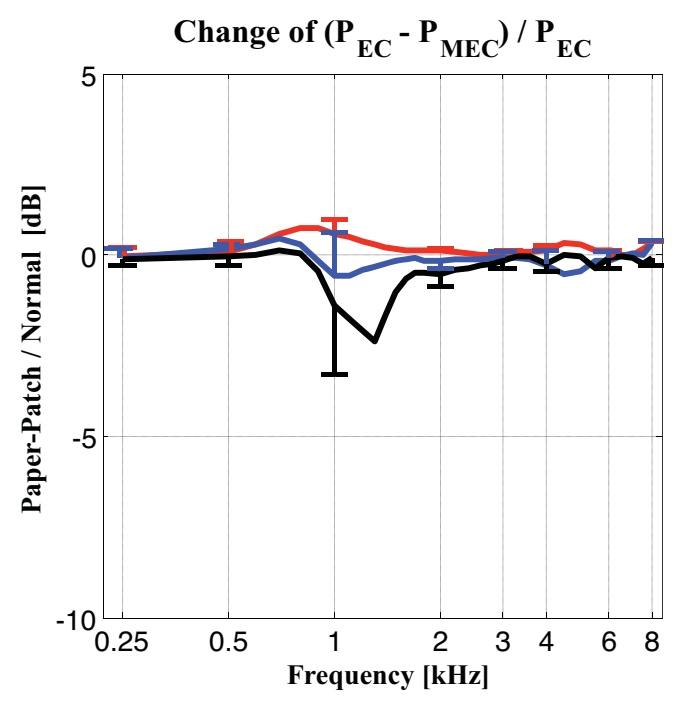

B

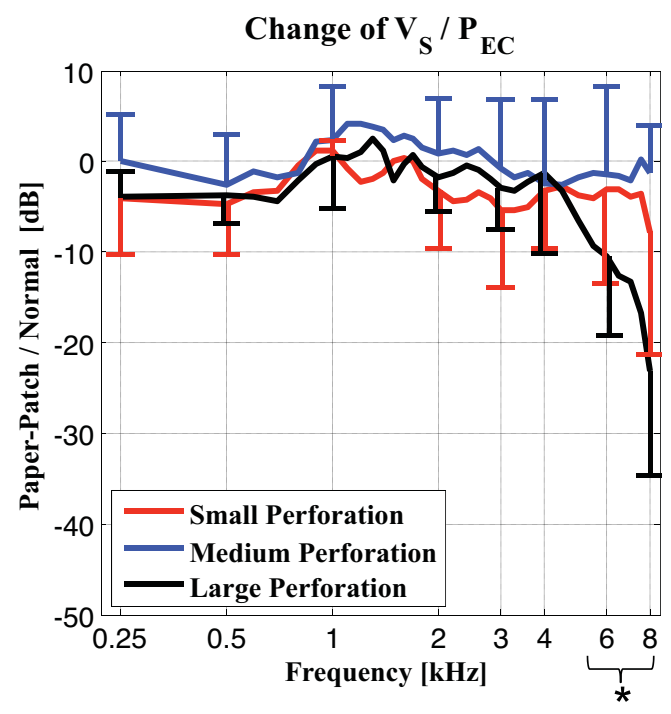


Figure 7

A

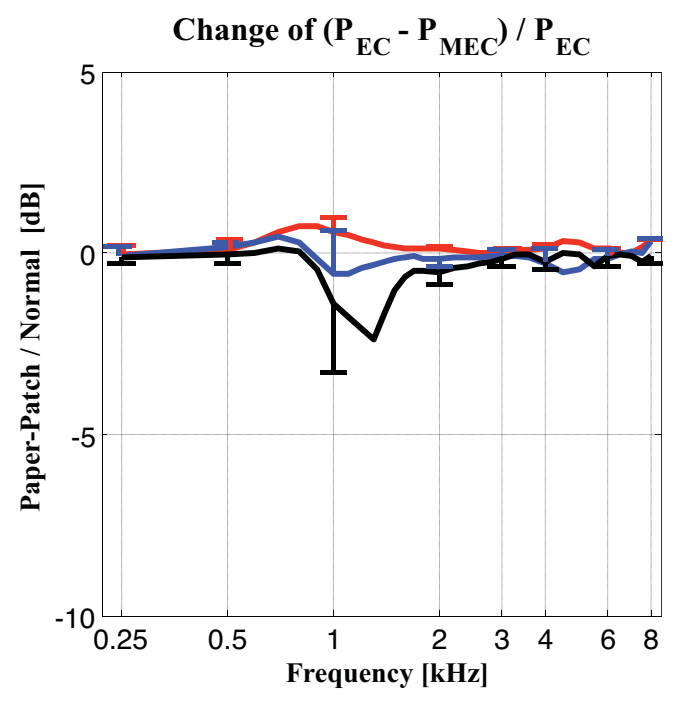

B

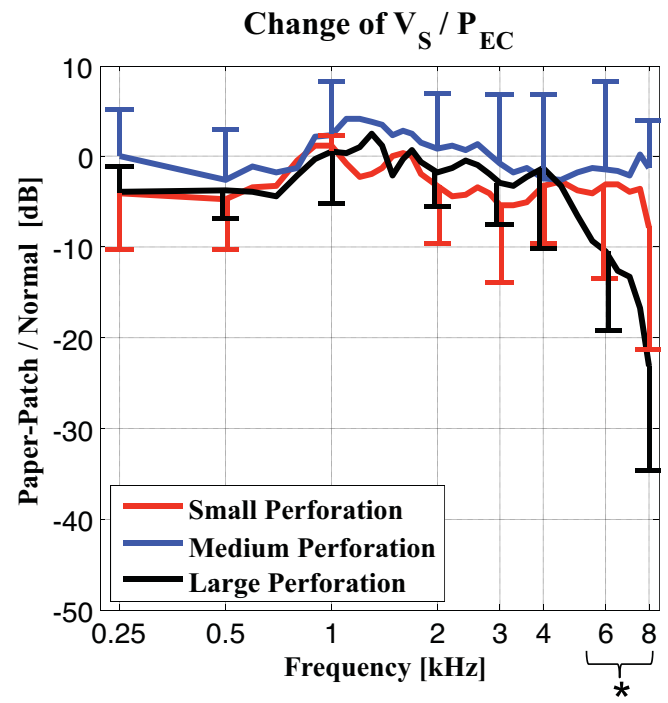

